\title{
Plant Growth Promotion and
} Induction of Systemic Tolerance to Drought and Salt Stress of Plants by Quorum Sensing Auto-Inducers of the $\mathbf{N}$-acyl-homoserine Lactone Type: Recent Developments

\author{
Anton Hartmann ${ }^{1 *}$, Sophia Klink ${ }^{2}$ and Michael Rothballer ${ }^{2}$ \\ ${ }^{1}$ Microbe-Host Interactions, Faculty of Biology, Ludwig-Maximilians-Universität München, Planegg, Germany, ${ }^{2}$ Institute \\ of Network Biology, Helmholtz Zentrum München, Neuherberg, Germany
}

OPEN ACCESS

Edited by:

Mariangela Hungria,

Embrapa Soja, Brazil

Reviewed by:

Francisco Javier Ollero,

University of Seville, Spain

Paula Cerezini,

Brazilian Agricultural Research Corporation (EMBRAPA), Brazil

*Correspondence:

Anton Hartmann

ahartmanndr@gmail.com

Specialty section:

This article was submitted to

Plant Abiotic Stress,

a section of the journal

Frontiers in Plant Science

Received: 21 March 2021

Accepted: 06 May 2021

Published: 31 May 2021

Citation:

Hartmann A, Klink S and

Rothballer M (2021) Plant Growth Promotion and Induction of Systemic Tolerance to Drought and Salt Stress

of Plants by Quorum Sensing

Auto-Inducers of the

$\mathrm{N}$-acyl-homoserine Lactone Type:

Recent Developments.

Front. Plant Sci. 12:683546.

doi: 10.3389/fpls.2021.683546
Keywords: $\mathrm{N}$-acyl-homoserine lactones, priming, induced systemic tolerance, ACC deaminase, auxins, osmolytes

\section{INTRODUCTION}

During the evolution of land plants, plant-associated microbiomes co-evolved and were integrated into prokaryote-eukaryote holobionts. Functions of these metagenomically organized plants are based on the gene expression of all holobiont participants. The constitution of the holobiont is dependent on specific signaling and perception events between the microbial and plant partners. Microbially produced plant hormones and a diversity of other metabolites have key roles in the process of interkingdom interaction of organisms. In association/symbiosis with plant beneficial bacteria, the ability of plants to cope with abiotic and biotic stresses is enhanced. As example for systemic effects reducing biotic stress, AHL-producing Serratia liquefaciens MG1 conferred biocontrol of the phytopathogenic fungus Alternaria alternata in tomato plants (Schuhegger et al., 2006). The down-regulation of the stress hormone ethylene by degradation of the precursor 1aminocyclopropane-1-carboxylate (ACC) with ACC deaminases (Glick, 2014) has a key role in the beneficial interaction of bacteria with plants under abiotic stress conditions. The ability of degrading the ethylene precursor ACC is frequently found in bacteria isolated from the rhizosphere of salt-tolerant plants (Jha et al., 2012; Suarez et al., 2015; Hrynkiewicz et al., 2019). Other mechanisms for the mitigation of salinity and drought stress in plants, like the involvement of IAA by rhizobacteria (Egamberdieva et al., 2017), antioxidants, extracellular polymeric substances (EPS) or volatile organics were recently reviewed by Kumar et al. (2020). However, the induction of systemic tolerance to abiotic stress conditions by the quorum sensing auto-inducers of $N$-acylhomoserine lactone-type (AHL) or AHL-producing rhizobacteria was not mentioned in this recent review and is generally not much recognized yet.

Since more than two decades, a very sensitive and effective communication system within bacterial populations is known, based on auto-inducers. In Gram-negative bacteria, molecules of the $\mathrm{N}$-acyl-homoserine lactone type with hydrophilic (short) and hydrophobic (long) $\mathrm{CH}$-chain are produced by luxI-type genes (Hartmann and Schikora, 2012; Rothballer et al., 2018). These small molecules are synthesized by the bacteria themselves but also by others in their vicinity depending on environmental conditions in the bacterial habitat. Their perception and subsequent 
concentration dependent transcriptional regulation mediated by luxR-type receptors allow to efficiently adapt gene expression to environmental changes. This makes the auto-inducer signaling an essential and evolutionary relevant tool for the efficiency of bacterial behavior (Hense and Schuster, 2015). Interkingdom signaling of bacteria with host plants based on AHL autoinducers was demonstrated in many Gram-negative rhizobacteria as reviewed by Schikora et al. (2016). The most recently observed role of AHLs in the improvement of abiotic stress tolerance of some plants deserves to be widened for more different crops to be finally applied in practical agriculture.

\section{ROLES OF PHYTOHORMONES AND OSMOLYTES IN SALT AND DROUGHT TOLERANCE OF PLANTS}

Plant hormones play an essential role in the regulation of responses at environmental stress situations such as drought or high salinity. It has been reviewed in detail, which different mechanisms are active in a wide number of diverse plant growth promoting rhizobacteria (PGPR) conferring drought and salt tolerance to crop plants (Vurukonda et al., 2016). In all living organisms the key physiological basis of salt and drought tolerance is the accumulation of so-called osmolytes. Important osmolytes are certain amino acids (especially proline), amines (glycine betaine or ectoine) and sugars, which have specific physicochemical properties to protect cellular protein structures and balance high osmotic pressure. Oxygen stress is also accompanying abiotic and biotic stress situations. Therefore, the oxygen radical scavenging enzymes are also very important in these situations. For example, the oxygen radical scavenging enzyme superoxide dismutase of Gluconacetobacter diazotrophicus PAL5 is obligatory for endophytic colonization of rice (Alqueres et al., 2013). Concerning the hormonal drought-stress responses in plants, abscisic acid (ABA) and other phytohormones, such as jasmonates and ethylene are involved. In addition, the auxin indole-3-acetic acid (IAA) in an optimal ratio to other phytohormones plays an integral part in plant adaptation to water stress. It is therefore of particular importance under abiotic stress conditions that root associated bacteria themselves produce and excrete auxins and other phytohormones or influence the phytohormone balance supporting plants which have too low auxin levels due to salt inhibition. The use of salt-tolerant phytohormone producing bacteria in combination with high osmolyte containing seaweeds is widely distributed and successfully applied to support crop growth in salt affected soils as reviewed by Nabti et al. (2015). Another approach to solve the lack of IAA in plants under salt stress is the inoculation with IAA-overproducing Ensifer meliloti conferring drought tolerance to Medicago sativa (Defez et al., 2017b). Inoculation with IAA-overproducing strains of endophytic Burkholderia cepacia strain RRE25 caused stimulated root development and improved nutrient use efficiency (especially of phosphate uptake) leading to increased plant growth (Singh et al., 2013). In endophytic diazotrophic bacteria Enterobacter cloacae RCA25 and Klebsiella variicola RCA26 overproduction of IAA upregulated nitrogen fixation in bacterial cultures and inoculated rice plants (Defez et al., 2017a). Azospirillum brasilense Cd mutants which overproduce IAA could be selected using the L-tryptophan toxic antimetabolite 5-fluor-tryptophan (Hartmann et al., 1983), leading to plants with increased root system at low nitrogen supply. Furthermore, using the proline antimetabolite 3-dehydroproline (DHP), A. brasilense Sp7 mutants with increased osmotolerance have been isolated (Hartmann et al., 1992). Concerning proline-overproducing strains, many salt-tolerant bacteria are already DHP-resistant. The screening for DHP-resistance was not yet systematically applied to yield strains with improved salt and drought tolerance.

\section{QUORUM SENSING AUTOINDUCER $N$-ACYL-HOMOSERINE LACTONES AND AHL-PRODUCING RHIZOBACTERIA STIMULATE PLANT GROWTH UNDER NORMAL AND ABIOTIC STRESS CONDITIONS}

The influence of AHLs on plant performance is dependent on the molecular structure of the AHL-signal. Treatment with water-soluble C4- and C6-homoserine lactones (HSL) changes in the phytohormone balance occurred in roots and shoots of Arabidopsis thaliana (von Rad et al., 2008). It was found that the presence of GCR1/GPA1 genes in the plant host mediates the stimulation of root growth (Liu et al., 2012). In barley plants, AHL-molecules applied in axenic model experiments with seedlings initiated a nitrous oxide (NO)-burst and $\mathrm{Ca}^{2+}$-fluxes in root cells as well as morphological changes in roots (Rankl et al., 2016). As shown in A. thaliana, calmodulin receptors were involved in primary root elongation stimulated by 3oxo-C6 HSL (Zhao et al., 2015). Furthermore, it was shown that ATMYB44 was involved in the enhanced elongation of primary roots (Zhao et al., 2016). Using several independent experimental approaches, it could be clearly proven that watersoluble AHLs are taken up by plant roots through the vascular system into the shoot by an energy-dependent process. However, this is only possible in plants, like Arabidopsis thaliana, wheat, or barley, which are devoid of AHL-degrading enzymes, like lactonases. In plant shoots, hydrophilic C4-, C6-, and C8HSLs influence the activities of enzymes with e.g., anti-oxidative capacity or xenobiotic phase II detoxifying enzymes improving stress tolerance (Goetz-Roesch et al., 2015).

Water-insoluble AHLs with long $\mathrm{CH}$-chains (e.g., C12- and C14-HSL) are also effective in modifying plant performance (Schenk and Schikora, 2015). Several lines of evidences point to the involvement of a specific receptor for waterinsoluble AHLs in plant cells and the activation of a specific signaling cascade. In barley it was demonstrated that the ability of AHL-priming of systemic resistance is a genetically determined property (Wehner et al., 2019). Salicylic acid (SA) and oxylipin 12-oxo-phytodienoic acid (cis-OPDA) are involved in the systemic response and priming (Schenk and Schikora, 2015; Shrestha et al., 2020). In addition, the 
activation of MAP-kinases MPK3 and MPK6 were enhanced and prolonged, along with the upregulation of defense-related transcription factors WRKY22 and WRKY29, glutathione-Stransferase GST6-gene and heat shock protein Hsp60-gene in Arabidopsis by a mixture of short- and long $\mathrm{CH}$-chain AHLs (Shrestha et al., 2019). AHL-producing Burkholderia graminis M12 and M14 strains could induce tolerance to salt stress in wild type and genetically engineered tomato plants (Barriuso et al., 2008). It was recently shown by Zhao et al. (2020) that AHL-enhanced tolerance to salt stress in Arabidopsis thaliana and wheat was linked to ABAdependent and ABA-independent signaling pathways and also the Salt Overly Sensitive (SOS) pathway. 3-oxo-C6-HSL treated plants had an increased proline and chlorophyll content, while lipid peroxidation, as measured by tissue content of malondialdehyde, and the $\mathrm{Na}^{+} / \mathrm{K}^{+}$ratio was decreased. It was clearly demonstrated that inoculation with AHL-producing plant beneficial Gram-negative bacteria like Gluconacetobacter diazotrophicus PAL5 supports plant growth under adverse conditions. For example, G. diazotrophicus mitigates drought stress in Oryza sativa (Filgueiras et al., 2020), resulting in higher biomass production, higher levels of gas exchange and osmoprotecting solutes (proline and glycine betaine) in shoots and roots under drought conditions. Under reduced water availability malondialdehyde accumulation, a product of lipid peroxidation, increased drastically as result of cell damages, but in PAL5-inoculated plants the accumulation of malondialdehyde was much reduced. In addition, the anti-oxidant enzymes superoxide dismutase $(\operatorname{sod} A)$, glutathione reductase (gor), catalase (katE), pyrrolinbe-5-carboxylate reductase (P5CR) and betaine aldehyde dehydrogenase $(B A D H)$ were stimulated by inoculation with $G$. diazotrophicus PAL5 under increased water stress. Interestingly, this "induced systemic tolerance" at reduced water availabilities was accompanied by an increased transcription of both pathogenesis related PR-1 and PR-10 genes, which are known to function in the salicylic acid (SA) and jasmonic acid (JA) pathways. Furthermore, in G. diazotrophicus PAL5 exposed to reduced water availability, AHL synthase gene expression increased dramatically, which is linked to a stimulation of $\mathrm{N}$-acyl-homoserine lactone production. Thus, AHLs are probably the key bacterial signals behind the induced systemic tolerance response in red rice, reported by Filgueiras et al. (2020).

\section{AHL-DEVOID MUTANTS ARE IMPAIRED IN BENEFICIAL PLANT EFFECTS}

The central role of AHLs in the plant beneficial effects was proven several times with mutants lacking the $\operatorname{luxI-}$ or $\operatorname{luxR}$ gene homologue or in AHL-depleted transconjugants of PGPR. Rhizobium radiobacter $\mathrm{F} 4$ has been first demonstrated as endofungal bacterium of the plant growth promoting fungus Serendipita indica (syn. Piriformospora indica) (Sharma et al., 2008; Varma et al., 2012). However, even without the fungus, $R$. radiobacter $\mathrm{F} 4(\mathrm{RrF} 4)$ proved to have very similar plant beneficial effects as $S$. indica, like improved abiotic and biotic stress tolerance (Glaeser et al., 2015). Recently, it was

\section{The presence of AHLs or AHL-producing bacteria initiate modifications on:}

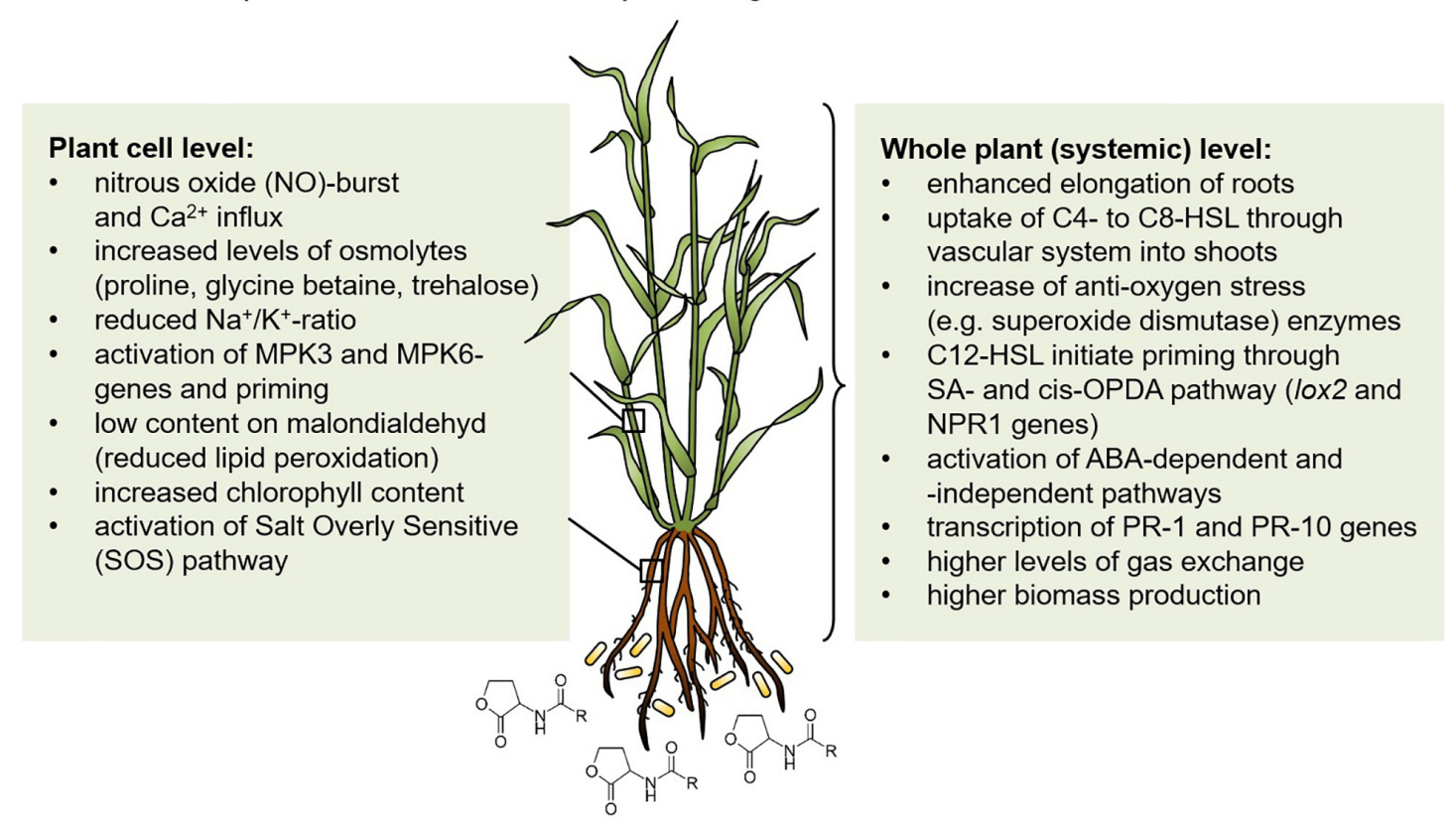

FIGURE 1 | Induced systemic tolerance against drought and salt stress in N-acyl-homoserine lactone-treated plants. MPK, Mitogen-associated protein kinase; SA, Salicylic acid; cis-OPDA, 12-oxo-phytodienoic acid; PR-1 and PR-10, Pathogenesis related proteins; ABA, Abscisic acid; lox, Lipoxygenase; NPR1, Nonexpressor of pathogenesis-related genes 1 . 
demonstrated that $R$. radiobacter $\mathrm{F} 4$ produced a spectrum of short and medium $\mathrm{CH}$-chain AHL molecules (Alabid et al., 2020). R. radiobacter transconjugants F4NM13 with a lactonaseproducing plasmid, which are devoid of AHLs, showed only reduced colonization abilities of roots of Arabidopsis thaliana and barley and only marginal plant beneficial effects. In the PGPR Acidovorax radicis N35 (Li, 2010), producing 3-OH-C10HSL, araI-deleted mutants were less effective in root colonization in competition with the wild type. While the AHL-producing wild type induced a plant expression profile of stimulation and some priming, the AHL-deleted mutant caused increased expression of defense responses like flavonoid biosynthesis (Han et al., 2016). Thus, in A. radicis, AHL-production could influence the perception by a host plant. This was also indicated by Arabidopsis thaliana and barley inoculation with the AHLnegative tranconjugant Rhizobium radiobacter F4NM13 (Alabid et al., 2020), but more studies are necessary to further document this important issue.

\section{POSSIBLE ROLES OF LUXR-SOLOS IN PLANT BENEFICIAL EFFECTS}

In the frequently applied Azospirillum brasilense strains AbV5, Sp245, Sp7, or Az39 and also in other Azospirillum species strains (Gualpa et al., 2019) luxR-solo or luxR-orphan genes were found, i.e., luxR-homologous receptors without the presence of a luxI-type AHL-synthase. It has been shown by Fukami et al. (2018) that the addition of 3-oxo-C6-HSL to luxR-solo A. brasilense Ab-V5 caused a significant stimulation of plant colonization traits, demonstrating that external available AHLs were perceived and resulted in a positive response of stimulated bacteria-plant interaction. It has been proposed by Patel et al. (2013) that bacterial luxR-solos have evolved to respond to AHL-related or even rather unrelated signals from plants. This evolution of the luxI-/luxR-type quorum sensing regulon may be regarded as the basis of a novel signal/receptor couple for plantbacteria communication (Schaefer et al., 2013, 2016). According to Gualpa et al. (2019), quorum quenching activities were prevalent in A. brasilense Az39, which also lacks a luxI-type AHLbiosynthesis gene. It may be possible, that A. brasilense Az39 has already gone steps forward in the evolution of the QS system and specialized on the degradation of AHLs. This may provide the bacterium an advantage to neutralize competing AHLproducing bacteria in the rhizosphere and in parallel facilitates receiving communication signals from the plant through $\operatorname{luxR}$ solo receptors.

\section{FUTURE PERSPECTIVES}

A high diversity of rhizobacteria support plants in environmental stress conditions, especially under salt and drought stress using different mechanisms (Kumar et al., 2020). In addition, QS signals of the $N$-acyl-homoserine lactone-type from Gramnegative bacteria stimulate plant development and prime systemic tolerance to environmental stress in crop plants. This stimulation occurs at cellular as well as whole plant level, as summarized in Figure 1. Thus, it should be a desirable goal to use AHL-producing bacteria or their AHL-signals as well as AHL quorum sensing related activities to stimulate plant tolerance to abiotic stress. In this context, synthetic biology would be a helpful approach (Stephens and Bentley, 2020). The entire network of interactions of the plant microbiome with host plants under different biotic and abiotic stress situations needs to be further developed also in a systems biology approach (Rodriguez et al., 2019). Besides the stimulatory interaction of AHL-producing bacteria with plants, C4- and C6-HSL molecules as foliar spray or seed treatment should be applied to improve plant performance, yields and resistance to biotic and abiotic stresses as was successfully demonstrated by Moshynets et al. (2019). Furthermore, a nanocomposite fertilizer using magnetic carbon nanofibers coupled with C4-HSL stimulated seed germination and growth of crops with enhanced resistance to oxidative and high salinity stresses (Gupta et al., 2019). Thus, the application of a synthetic AHL concentrate for bacteriaplant stimulation started already quite promising and AHL-QS research should be emphasized in the future involving more different crop plants. However, it should be kept in mind that not all cultivars are susceptible for AHL-perception (Shrestha et al., 2019). More basic understanding of the underlying mechanisms should further support successful field applications to modify and improve crop growth in abiotic and biotic challenging conditions.

\section{AUTHOR CONTRIBUTIONS}

$\mathrm{AH}$ designed the structure and content of the manuscript. SK wrote part of the manuscript, corrected the final manuscript, and contributed the figure. MR wrote part of the manuscript and corrected the final manuscript. All authors contributed to the article and approved the submitted version.

\section{FUNDING}

Deutsche Forschungsgemeinschaft (DFG) Grant No. RO2340/41 supported SK in the ongoing project. We thank Helmholtz Zentrum München for institutional funding of the former Research Unit Microbe-Plant Interactions, headed by $\mathrm{AH}$, the special inhouse funding of the interdisciplinary research project "Molecular signaling in the Rhizosphere" and the current Institute of Network biology (MR). The DFG-project Ro2340/4-1 covers the fees for open access publication.

\section{ACKNOWLEDGEMENTS}

The support of Helmholtz Center Munich, German Research Center for Health and Environment, München/Neuherberg, during the interdisciplinary in house funding topic "Molecular Interactions in the Rhizosphere" is greatly appreciated. The contribution of Sophia Klink to this manuscript was supported by Deutsche Forchungsgemeinschaft (DFG), grant RO 2340/4-1. 


\section{REFERENCES}

Alabid, I., Hardt, M., Imani, J., Hartmann, A., Rothballer, M., Li, D., et al. (2020). The $N$-acyl-homoserine lactone depleted Rhizobium radiobacter RrF4NM13 shows reduced growth-promoting and resistance-inducing activities in monoand dicotyledonous plants. J. Plant Dis. Prot. 8:127. doi: 10.1007/s41348-02000360-8

Alqueres, S., Meneses, C., Rouws, L. M., Rothballer, M., Baldani, I., Schmid, M., et al. (2013). The bacterial superoxide dismutase and glutathione reductase are crucial for endophytic colonization of rice roots by Gluconacetobacter diazotrophicus PAL5. Mol. Plant Microbe Interact. 26, 937-945. doi: 10.1094/ mpmi-12-12-0286-r

Barriuso, J., Solano, B. R., Fray, R. G., Cámara, M., Hartmann, A., and Gutierrez Manero, F. J. (2008). Transgenic tomato plants alter quorum sensing in plant growth- promoting rhizobacteria. Plant Biotech. J. 6, 442-452. doi: 10.1111/j. 1467-7652.2008.00331.x

Defez, R., Andreozzi, A., and Bianco, C. (2017a). Overproduction of IAA in endophytes upregulate nitrogen fixation in both bacterial cultures and inoculated rice plants. Microb. Ecol. 74, 441-452. doi: 10.1007/s00248-0170948-4

Defez, R., Andreozzi, A., Dickinson, M., Charlton, A., Tadini, L., Pesaresi, P., et al. (2017b). Improved drought stress response in Alfalfa plants nodulated by an IAA- overproducing Rhizobium strain. Front. Microbiol. 8:e2466. doi: 10.3389/fmicb.2017.02466

Egamberdieva, D., Wirth, S. J., Alqarawi, A. A., Abd Allah, E. F., and Hashem, A. (2017). Phytohormones and beneficial microbes: essential components for plants to balance stress and fitness. Front. Microbiol. 8:2104. doi: 10.3389/fmicb. 2017.02104

Filgueiras, L., Silva, R., Almeida, I., Vidal, M., Baldani, J. I., and Menesses, C. H. S. (2020). Gluconacetobacter diazotrophicus mitigates drought stress in Oryza sativa L. Plant Soil 451, 57-73. doi: 10.1007/s11104-019-04163-1

Fukami, J., Abrantes, J. L. F., del Cerro, P., Nogueira, M. A., Ollero, F. J., Megias, M., et al. (2018). Revealing strategies of quorum sensing in Azospirillum brasilense strains Ab-V5 and Ab-V6. Arch. Microbiol. 200, 47-56. doi: 10.1007/s00203017-1422-x

Glaeser, S. P., Imani, J., Alabid, I., Guo, H., Kämpfer, P., Rothballer, M., et al. (2015). The endofungal bacterium Rhizobium radiobacter $\mathrm{RrF} 4$ colonizes plant roots and exhibits beneficial activity independently of its fungal host Piriformospora indica. ISME J. 10, 871-884. doi: 10.1038/ismej.2015.163

Glick, B. R. (2014). Bacteria with ACC deaminase can promote plant growth and help to feed the world. Microbiol. Res. 169, 30-39. doi: 10.1017/j.micres.2013. 09.009

Goetz-Roesch, C., Riedel, T., Schmitt-Kopplin, P., Hartmann, A., and Schroeder, P. (2015). Influence of bacterial $N$-acyl-homoserine lactones on growth parameters, pigments, anti-oxidative capacities and the xenobiotic phase II detoxification enzymes in barley and yam bean. Front. Plant Sci. 6:205. doi: 10.3389/fpls.2015.00205

Gualpa, J., Lopez, G., Nievas, S., Coniglio, A., Halliday, N., Cámara, M., et al. (2019). Azospirillum brasilense Az39, a model rhizobacterium with AHL quorum-quenching capacity. J. Appl. Microbiol. 14:269. doi: 10.1111/jam.14269

Gupta, G. S., Kumar, A., and Verma, N. (2019). Bacterial homoserine lactones as a nanocomposited fertilizer and defense regulator for chickpeas. Environ. Sci. Nano. 6, 1246-1258. doi: 10.1039/c9en00199a

Han, S., Li, D., Trost, E., Mayer, K. F., Vlot, C., et al. (2016). Systemic response of barley to the 3-hydroxy-decanoyl-homoserine lactone producing plant beneficial endophyte Acidovorax radicis N35. Front. Plant Sci. 7:e1868. doi: $10.3389 /$ fpls. 2016.01868

Hartmann, A., Gündisch, C., and Bode, W. (1992). Azospirillum mutants improved in iron aquisition and osmotolerance as tools for the investigation of environmental fitness traits. Symbiosis 13, 271-279.

Hartmann, A., and Schikora, A. (2012). Quorum sensing of bacteria and transkingdom Interactions of $\mathrm{N}$-acyl homoserine lactones with eukaryotes. J. Chem. Ecol. 38, 704-713. doi: 10.1007/s10886-012-0141-7

Hartmann, A., Singh, M., and Klingmüller, W. (1983). Isolation and characterization of Azospirillum mutants excreting high amounts of indole acetic acid. Can. J. Microbiol. 29, 916-923. doi: 10.1139/m83-147

Hense, B. A., and Schuster, M. (2015). Core principles of bacterial auto-inducer systems. Microbiol. Mol. Biol. Rev. 79, 153-169. doi: 10.1128/MMBR.00024-14
Hrynkiewicz, K., Patz, S., and Ruppel, S. (2019). Salicornia europaea L. as an underutilized saline-tolerant plant inhabited by endophytic diazotrophs. J. Adv. Res. 19, 49-56. doi: 10.1016/j.jare.2019.05.002

Jha, B., Gontia, I., and Hartmann, A. (2012). The roots of the halophyte Salicornia brachiata are source of new halotolerant diazotrophic bacteria with plant growth promoting potential. Plant Soil 356, 265-277. doi: 10.1007/s11104-0110877-9

Kumar, A., Singh, S., Gaurav, A. K., Srivastava, S., and Verma, J. P. (2020). Plant growth-promoting bacteria: biological tools for the mitigation of salinity stress in plants. Front. Microbiol. 11:e1216. doi: 10.3389/fmicb.2020. 01216

Li, D. (2010). Phenotypic variation and molecular signalling in the interactions of the rhizosphere bacteria Acidovorax radicis $\mathrm{N} 35$ and Rhizobium radiobacter $\mathrm{F} 4$ with roots. $\mathrm{Ph} \mathrm{D}$ thesis, Germany: Ludwig-Maximilians-Universität München.

Liu, F., Bian, Z., Jia, Z., Zhao, Q., and Song, S. (2012). The GCR1 and GPA1 participate in promotion of $A$. thaliana primary root elongation induced by $N$-acyl-homoserine lactones, the bacterial QS-signals. Mol. Plant Microbe Interact. 25, 677-683. doi: 10.1094/MPMI-10-11-0274

Moshynets, O. V., Babenko, L. M., Rogalsky, S. P., Iungin, O. S., Foster, J., Kosakivaska, I. V., et al. (2019). Priming winter wheat seeds with the bacterial quorum sensing $N$-hexanoyl-L-homoserine lactone (C6-HSL) shows potential to improve plant growth and seed yield. PLoS One 14:e0209460. doi: 10.1371/ journal.pone.0209460

Nabti, E., Schmid, M., and Hartmann, A. (2015). Application Of Halotolerant Bacteria To Restore Plant Growth Under Salt Stress. Halophiles: Springer-Verlag, 235-259.

Patel, H. K., Suárez-Moreno, Z. R., Degrassi, G., Subramoni, S., González, J. F., and Venturi, V. (2013). Bacterial LuxR solos have evolved to respond to different molecules including signals from plants. Front. Plant Sci. 4:e447. doi: 10.3389/ fpls.2013.00447.03

Rankl, S., Gunsé, B., Sieper, T., Schmid, C., Poschenrieder, C., and Schröder, P. (2016). Microbial homoserine lactones (AHLs) are effectors of root morphoplogical changes in barley. Plant Sci. 253, 130-140. doi: 10.1016/j. plantsci.2016.09.014

Rodriguez, P. A., Rothballer, M., Chowdhury, S. P., Nussbaumer, T., Gutjahr, C., and Falter-Braun, P. (2019). Systems Biology of PlantMicrobiome Interactions. Molec. Plant 12, 804-821. doi: 10.1016/j.molp.2019. 05.006

Rothballer, M., Uhl, J., Kunze, J., Schmitt-Kopplin, P., and Hartmann, A. (2018). "Detection of bacterial quorum sensing signaling molecules $\mathrm{N}$-acyl-homoserine lactones and $N$-acyl homoserine with an enzyme-linked immunosorbent assay (ELISA) and via ultrahigh performance liquid chromatography coupled to mass spectrometry (UPLC-MS)," in Quorum Sensing: Methods and Protocols. Springer series "Methods in Molecular Biology", eds L. Leoni and G. Rampioni (Netherland: Springer Science), 61-72. doi: 10.1007/978-1-4939-73 09-5_5

Schaefer, A. L., Lappala, C. R., Morlen, R. P., Pelletier, D. A., Lu, T. S., Lankford, P. K., et al. (2013). LuxR- and LuxI-type quorum sensing circuits are prevalent in members of the Populus deltoides microbiome. Appl. Environ. Microbiol. 79, 5745-5752. doi: 10.1128/AEM.01417-13

Schaefer, A. L., Oda, Y., Coutinho, B. G., Pelletier, D. A., Weiburg, J., Venturi, V., et al. (2016). A $\operatorname{luxR}$ homolog in a cottonwood tree endophyte that activates gene expression in response to a plant signal or specific peptides. mBio 7 , 1101-1116e. doi: 10.1128/mBio.01101-16

Schenk, S. T., and Schikora, A. (2015). AHL-priming functions via oxylipin and salicyclic acid. Front. Plant Sci. 5:e784.

Schikora, A., Schenk, S. T., and Hartmann, A. (2016). Beneficial effects of bacteriaplant communication based on quorum sensing molecules of the $\mathrm{N}$-acylhomoserine lactone group. Plant Mol. Biol. 90, 605-612. doi: 10.1007/s11103016-0457-8

Schuhegger, R., Ihring, A., Gantner, S., Bahnweg, G., Knappe, C., Vogg, G., et al. (2006). Induction of systemic resistance in tomato plants by $N$-acyl-homoserine lactone-producing rhizosphere bacteria. Plant Cell Environ. 29, 909-918. doi: 10.1111/j.1365-3040.2005.01471.x

Sharma, M., Schmid, M., Rothballer, M., Hause, G., Zuccaro, A., Imani, J., et al. (2008). Detection and identification of mycorrhiza helper bacteria intimately associated with representatives of the order Sebacinales. Cell. Microbiol. 10, 2235-2246. doi: 10.111/j.1462-5822.2008.01202.x 
Shrestha, A., Elhady, A., Adss, S., Wehner, G., Böttcher, C., Heuer, H., et al. (2019). Genetic differences in barley govern the responsiveness to $\mathrm{N}$-acyl homoserine lactone. Phytobiomes J. 3, 191-202. doi: 10.1094/pbiomes-03-19-0015-r

Shrestha, A., Grimm, M., Ojiro, I., Krumweide, J., and Schikora, A. (2020). Impact of quorum sensing molecules on plant growth and immune system. Front. Microbiol. 11:e1545.

Singh, R. K., Malik, N., and Singh, S. (2013). Improved nutrient use efficiency increases plant growth of rice with the use of IAA-overproducing strains of endophytic Burkholderia cepacia strain RRE25. Microb. Ecol. 66, 375-384. doi: 10.1007/s00248-013-0231-2

Stephens, K., and Bentley, W. E. (2020). Synthetic biology for manipulating quorum sensing in microbial consortia. Trends Microbiol. 2, 633-643. doi: 10.1016/j.tim.2020.03.009

Suarez, C., Cardinale, M., Ratering, S., Steffens, D., Jung, S., Montoya, A. M. Z., et al. (2015). Plant growth promoting effects of Hartmannibacter diazotrophicus on summer barley (Hordeum vulgare, L.) under salt stress. Appl. Soil. Ecol. 95, 23-30. doi: 10.1016/j.apsoil.2015.04.017

Varma, A., Bakshi, M., Lou, B., Hartmann, A., and Oelmueller, R. (2012). Piriformospora indica: A novel plant growth-promoting mycorrhizal fungus. Agric. Res. 1, 117-131. doi: 10.1007/s40003-012-0019-5

von Rad, U., Klein, I., Dobrev, P. I., Kottova, J., Zazimalova, E., Fekete, A., et al. (2008). The response of Arabidopsis thaliana to $N$ - hexanoyl-DL-homoserinelactone, a bacterial quorum sensing molecule produced in the rhizosphere. Planta 229, 73-85. doi: 10.1007/s00425-008-0811-4

Vurukonda, S. S. K. P., Vardharajula, S., Shrivastava, M., and Skz, A. (2016). Enhancement of drought stress tolerance in crops by plant growth promoting rhizobacteria. Microbiol. Res. 184, 13-24. doi: 10.1016/j.micres.2015.12.003
Wehner, G., Kopalmke, D., Richter, K., Kecke, S., Schikora, A., and Ordon, F. (2019). Priming is a suitable strategy to enhance resistance towards leaf rust in barley. Phytobiome J. 18:41. doi: 10.1094/PBIOMES-09-18-0041-R

Zhao, Q., Li, M., Jia, Z., Liu, F., Ma, H., Huang, Y., et al. (2016). AtMYB44 positively regulates the enhanced elongation of primiary roots induced by $\mathrm{N}$-oxo-hexanoyl homoserine lactone in Arabidosis thaliana. Mol. Plant Microbe Interact. 29, 774-785. doi: 10.1094/mpmi-03-160063-r

Zhao, Q., Yang, X. Y., Li, Y., Liu, F., Cao, X. Y., Jia, Z. H., et al. (2020). N-3-oxohexanoyl-homoserine lactone, a bacterial quorum sensing signal, enhances salt tolerance in Arabidopsis and wheat. Bot. Stud. 61:8. doi: 10.1186/s40529-02000283-5

Zhao, Q., Zhang, C., Jia, Z., Huang, Y., Li, H., Song, S., et al. (2015). Involvement of calmodulin in regulation of primary root elongation by $\mathrm{N}$-3-oxo-hexanoyl homoserine lactone in Arabidopsis thaliana. Front. Plant Sci. 5:807. doi: 10. 3389/fpls.2014.00807

Conflict of Interest: The authors declare that the research was conducted in the absence of any commercial or financial relationships that could be construed as a potential conflict of interest.

Copyright (®) 2021 Hartmann, Klink and Rothballer. This is an open-access article distributed under the terms of the Creative Commons Attribution License (CC BY). The use, distribution or reproduction in other forums is permitted, provided the original author(s) and the copyright owner(s) are credited and that the original publication in this journal is cited, in accordance with accepted academic practice. No use, distribution or reproduction is permitted which does not comply with these terms. 